\title{
PERFORMANCE EVALUATION OF SEQUENTIAL BAND ORIENTATION BY POLYNOMIAL MODELS IN HYPERSPECTRAL CUBES COLLECTED WITH UAV
}

\author{
A. Berveglieri ${ }^{1,}$ *, A. M. G. Tommaselli ${ }^{2}$, G. Santos $^{3}$, L. D. Santos ${ }^{3}$, E. Honkavaara ${ }^{4}$ \\ ${ }^{1}$ Department of Statistics, São Paulo State University, Pres. Prudente, S.P., Brazil - a.berveglieri@unesp.br \\ ${ }^{2}$ Department of Cartography, São Paulo State University, Pres. Prudente, S.P., Brazil - a.tommaselli@ unesp.br \\ ${ }^{3}$ Graduate Student, São Paulo State University, Pres. Prudente, S.P., Brazil - guilherhsantos@gmail.com, \\ dias_lucas_santos@hotmail.com \\ ${ }^{4}$ Department of Remote Sensing and Photogrammetry, Finnish Geospatial Research Institute FGI, Finland - eija.honkavaara@ nls.fi
}

Commission I, WG I/9

KEY WORDS: Hyperspectral frame camera, Image orientation, Photogrammetry, Polynomial model, UAV

\begin{abstract}
:
A study on orientation of hyperspectral band cubes acquired with frame camera is presented in this paper. The camera technology is based on a tuneable Fabry-Perot Interferometer (FPI) and captures cubes of images sequentially using two sensors. However, the bands are not recorded at the same instant, which results different exterior orientation parameters (EOPs) for each image band. A technique based on polynomial model is assessed, which determines the EOPs within the hypercube from few sample bands, since a large number of bands are generated. Experiments were performed to assess the feasibility of using the polynomial technique. An analysis of the UAV trajectory was performed and the results of the polynomial technique were compared with those obtained by a conventional bundle adjustment. The trials showed that the results of both techniques were comparable, indicating that the time-dependent polynomial model can be used to estimate the EOPs of all spectral bands, without requiring a bundle adjustment including all bands. The accuracy of the block adjustment was analysed based on the discrepancies obtained from independent checkpoints. The root mean square error (RMSE) was calculated and showed an accuracy of approximately 1 GSD in planimetry and 1.5 GSD in altimetry. This accurate result is important because the proposed technique can significantly reduce the processing workload.
\end{abstract}

\section{INTRODUCTION}

This paper presents an experimental assessment on the orientation of a hyperspectral sensor based on the time-sequential acquisition principle. Such sensor is a Rikola camera model that uses a tuneable Fabry-Perot Interferometer (FPI) and acquires non-synchronized hyperspectral images in frame format (Saari et al., 2011). The sensor is lightweight, has small dimensions and can be loaded by UAVs for several types of photogrammetric and remote sensing applications, such as in forestry, agriculture, water and natural resources management. Several examples of applications using lightweight hyperspectral sensors in UAVs can be found, such as the works developed by Aasen et al. (2015), Honkavaara et al. (2017), Nevalainen et al. (2017), Oliveira et al. (2019), among others. As advantages, monitoring tasks and more detailed studies can be performed due to the high spatial, spectral and temporal resolutions.

In this type of sensor that uses sequential image acquisition, a problem to be solved is a more efficient technique for determination of the exterior orientation parameters (EOPs). When image acquisitions are made in mobile applications, each spectral band has different EOPs due to platform movement, that is, the acquisition results in a non-registered band sequence in the hypercube, following the platform movement. Another important feature of this camera is the use of two sensors. One of them captures visible bands $(506-636 \mathrm{~nm})$ while the other collects visible and NIR bands $(650-820 \mathrm{~nm})$. The number of spectral bands is defined by the user according to the type of application.
When working with spectral mapping using high resolution images, accurate interior and exterior orientations are required. Thus, EOPs must be accurately estimated to enable a spectral ortho-mosaic generation with high quality. The standard image orientation procedure is usually based on bundle block adjustment, but this requires using all bands. Alternatively, we have developed a polynomial-based approach to model the band displacement within each cube (Berveglieri et al., 2017). The main objective is to assess the quality of EOPs estimated by the polynomial technique and the EOP behaviour in the UAV trajectory. Since hyperspectral cubes have multiple bands, the polynomial technique uses a minimum number of bands as reference to estimate polynomial model parameters. Later, EOPs of all bands can be interpolated without requiring a bundle adjustment with all bands, which would be complicated due to the large number of image cubes (thousands of cubes) that can result from an aerial survey. Furthermore, control points would also be needed to be measured on a large number of spectral bands, which would be an exhaustive task.

Previous works with this technique were done with simulations considering a single sensor (Berveglieri et al., 2017). For this work, we have assembled a quadcopter UAV with the hyperspectral camera connected to a GPS receiver to determine positional data and GPS time. A second receiver recording raw data (L1-GPS) was also used. Therefore, experiments with real data were performed to evaluate the proposed technique and will be presented in the next sections.

\footnotetext{
* Corresponding author
} 


\section{BACKGROUND}

\subsection{Hyperspectral camera}

The camera used for the experiments is presented in Figure 1. This camera is a Rikola model (Senop Ltd., 2017) composed of two CMOS sensors that redirect the rays by a beam splitting device. The camera is also equipped with an irradiance sensor and a GPS receiver, which provides the GPS time of the first band of each hypercube and geographic coordinates (latitude and longitude). The positions of the subsequent bands within the hypercube are calculated based on the time delay for each band using a spreadsheet provided by the manufacturer. Table I presents the main technical details about the hyperspectral camera.
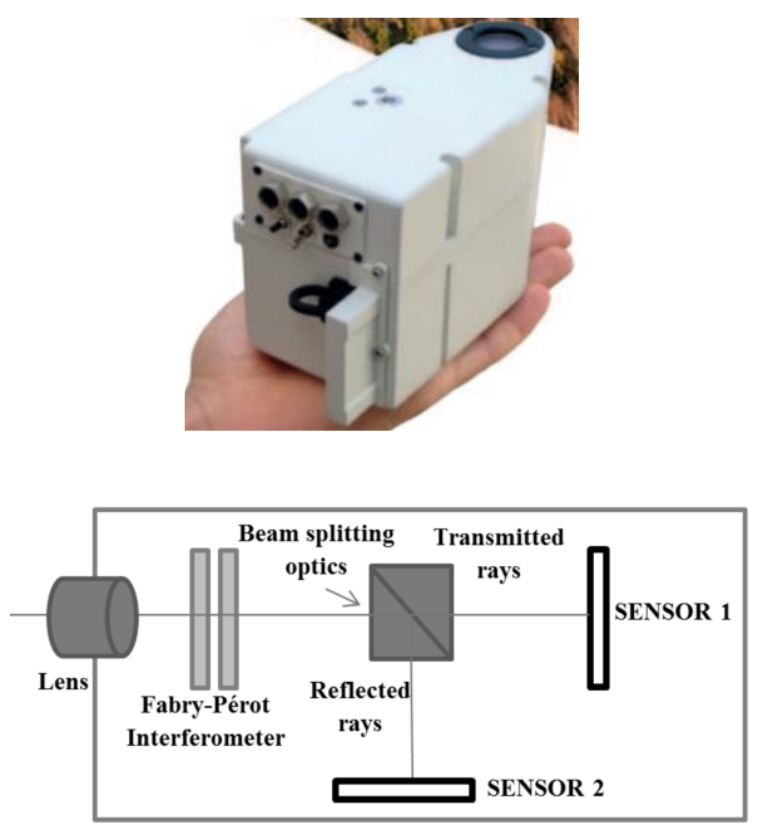

Figure 1. Rikola camera model and a sketch representing its internal system. Source: Oliveira et al. (2016).

\begin{tabular}{|l|l|}
\hline Camera model & Rikola FPI2015 \\
\hline Nominal focal length & $9 \mathrm{~mm}$ \\
\hline Pixel size & $5.5 \mu \mathrm{m}$ \\
\hline Image dimension & $1017 \times 648$ \\
\hline Sensors & $2 \mathrm{CMOS}$ \\
\hline Spectral resolution & $\begin{array}{l}10 \mathrm{~nm}, \text { FWHM (full width at half } \\
\text { maximum) }\end{array}$ \\
\hline Weight & $\sim 700 \mathrm{~g}$ \\
\hline
\end{tabular}

Table 1. Technical features of the Rikola camera.

Using several air gap values for the FPI, a set of wavelengths [500-900 nm]. can be produced with the two sensors. In this type of camera, the spectral bands are not captured at the same instant, resulting in different band positions during the aerial flight. Another important technical feature is that the two sensors are not perfectly aligned. Due to this misalignment, boresight angles have to be calculated to estimate the EOPs of bands sequence within the cube continuously. Such boresight angles are important for both the estimation of IOPs and the determination of EOPs by polynomial models.

\subsection{Polynomial models}

The EOPs of the spectral bands within each hypercube vary continuously as a function of time. Thus, if the UAV platform is moving, the band displacements in the direction of the flight trajectory can be modelled by polynomial functions, as represented in Eq. (1). A set of second-order polynomials is used to model the $(X, Y, Z)$ and attitude $(\omega, \varphi, \kappa)$ of each spectral band, in which the parameters $\left(a_{i}\right)$ model the acceleration and $\left(b_{i}\right)$ model the platform speed for each component of the platform, and $\left(\mathrm{c}_{\mathrm{i}}\right)$ represents initial position and attitude of the first band. The time interval $t$ of each band, with respect to the first band, is provided by the configuration software developed by Senop Ltd. (2017).

$$
f(t)=a_{i} t^{2}+b_{i} t+c_{i}
$$

The approach in this study proposes to use polynomial models intgrated with bundle adjustment to estimate the polynomial parameters of speed and acceleration by least squares method. Since computing all bands in a block of hypercubes is an extremely exhaustive and time-consuming task, the approach only uses four sample bands (two bands of each sensor) to determine the parameters of the time-dependent polynomial functions. Then, the EOPs to other bands within the hypercubes can be determined based on the estimated parameters (see section $3.3)$.

\section{DATA AND EXPERIMENTS}

\subsection{Camera calibration and boresight angles}

The inner orientation parameters (IOPs) of the Rikola camera were determined in a terrestrial calibration field, which is composed of coded targets with Aruco pattern (Garrido-Jurado et al., 2014). These targets were automatically identified and recognized to extract their corners to be used as ground control in the camera calibration procedure. A set of twelve hypercubes was taken from three different camera stations. Figure 2 shows an example of images taken in the 3D camera calibration field.

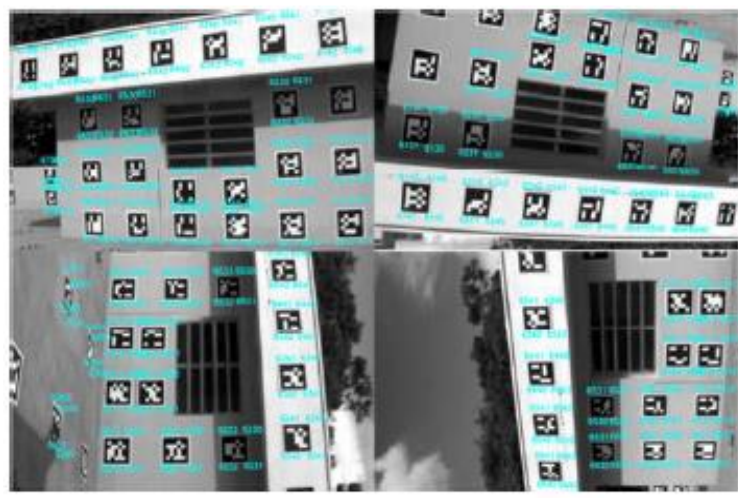

Figure 2. Examples of hyperspectral images used in the camera calibration.

For these experiments, the camera was configured with 25 bands, the visible sensor having 10 bands and the VNIR sensor with 15 bands. Then, a set of IOPs (focal length, principal distance and 
lens distortions) for each sensor was estimated using selfcalibrating bundle adjustment with constraints imposed to the ground coordinates, as proposed (Kenefick et al., 1972). The mathematical model was based on collinearity equations including additional parameters of the Conrady-Brown model (Fryer and Brown, 1986). A detailed study on the procedures used for camera calibration is given by Oliveira et al. (2016).

The boresight angles were estimated using a single hypercube. After determining the IOPs, ground coordinates and EOPs of all bands by the self-calibration procedure, the angles of the first ten bands of one sensor were compared to the angles of the ten bands of the other sensor (pairwise: $1^{\text {st }}$ band sensor 1 with $1^{\text {st }}$ band sensor $2,2^{\text {nd }} \times 2^{\text {nd }}, 3^{\text {rd }} \times 3^{\text {rd }}, \ldots$ ). The pair with the lowest standard deviations of EOPs was selected. The multiplication of the rotation matrix of one sensor by the transposed rotation matrix of the other allowed to calculate the boresight angles, as presented by Tommaselli et al. (2013).

\subsection{Image acquisition}

A UAV platform (Figure 3) was assembled to collect hyperspectral images using the Rikola camera, which is accompanied by a navigation GPS receiver. In addition, a singlefrequency GPS receiver (NavSpark) was included in the platform to acquire GPS raw data from which more accurate positions can be estimated.

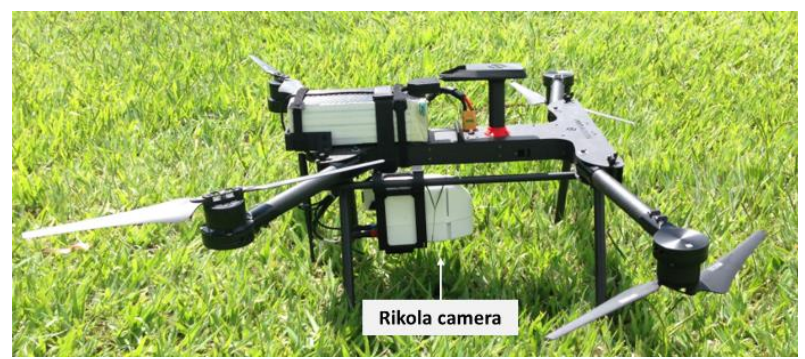

Figure 3. UAV platform with the Rikola hyperspectral camera.

Hyperspectral aerial images were acquired at a flying height of $160 \mathrm{~m}$ with flight speed of $4 \mathrm{~m} / \mathrm{s}$ over a study area with signalized GCPs and checkpoints. Thirty-seven hypercubes were captured in two flight strips for these trials. This flight configuration produced a forward overlap of $80 \%$ and side overlap of $60 \%$ and also generated images with ground sample distance (GSD) of $10 \mathrm{~cm}$.

\subsection{Hyperspectral band orientation}

A set of experiments was conducted with the polynomial models to assess the EOP behaviour using two sources of initial EOPs for image orientation: NavSpark positioning and navigation data from the GPS receiver attached to the Rikola camera. For purposes of comparison, the hyperspectral band orientation was performed with four sample bands using the polynomial models and with all 25 bands using a conventional bundle adjustment.

The photogrammetric block was configured with initial approximations for the EOPs and weighted constraints. The attitude angles were estimated as unknowns. A standard deviation of 1 pixel was assigned to the image coordinates due to the image blurring, whereas a standard deviation of $5 \mathrm{~cm}$ was used in the ground coordinates of the control points. The previously calibrated IOPs were configured as fixed in the adjustment procedure, since the boresight correction and the image coordinate refinement were performed before the image orientation procedure. Figure 4 shows the photogrammetric block used for the experiments with the bundle adjustment including the 25 bands, in which tie point were generated by the PhotoScan Agisoft.

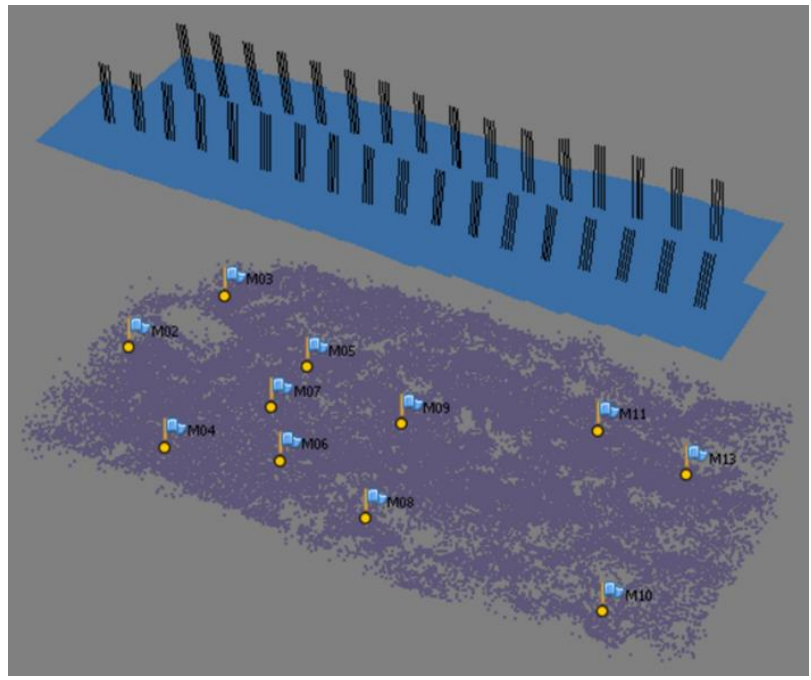

Figure 4. Photogrammetric block showing the flight strips, ground points and tie point cloud.

The time-dependent polynomial models were implemented and added to an in-house developed software (Marcato Junior and Tommaselli, 2013). Thus, four reference bands (1 and 10 of the visible sensor and 14 and 25 of the VNIR sensor) were used in the polynomial technique to estimate the parameters $a_{i}$ and $b_{i}$. The initial values for the speed parameters in $\mathrm{X}$ and $\mathrm{Y}$ were set based on the platform speed decomposed into XY components $(1.9 \mathrm{~m} / \mathrm{s}$ and $3.1 \mathrm{~m} / \mathrm{s}$ according to the flight direction). The other polynomial parameters were set to zero values. With this data set, the image orientations were performed and the results will be presented in the next section.

\section{RESULTS AND DISCUSSION}

As described in the previous section, two sets of initial camera positions were considered for the image orientation procedure (navigation data and NavSpark data) along with seven GCPs and four checkpoints. Accuracy assessments were based on discrepancies calculated from checkpoints and differences between positions acquired with the NavSpark receiver and those generated by image orientation techniques.

Figure 5 shows the root mean square error (RMSE) resulting at checkpoints when the image orientation was performed with initial camera positions provided by navigation data. For comparison with the polynomial technique, the image orientation was also performed by conventional bundle adjustment with 4 and 25 bands. In this case, the bundle adjustment trials with 4 and 25 bands obtained a better performance than with the polynomial technique, the RMSEs of the two bundle adjustments being close to the GSD level. Due to the inaccuracy of navigation data, the polynomial modelling achieved a discrepancy of $19.6 \mathrm{~cm}(\sim 2$ GSD) in X, $9.2 \mathrm{~cm}(\sim 1 \mathrm{GSD})$ in $\mathrm{Y}$ and $32.3 \mathrm{~cm}(\sim 3 \mathrm{GSD})$ in $\mathrm{Z}$. 


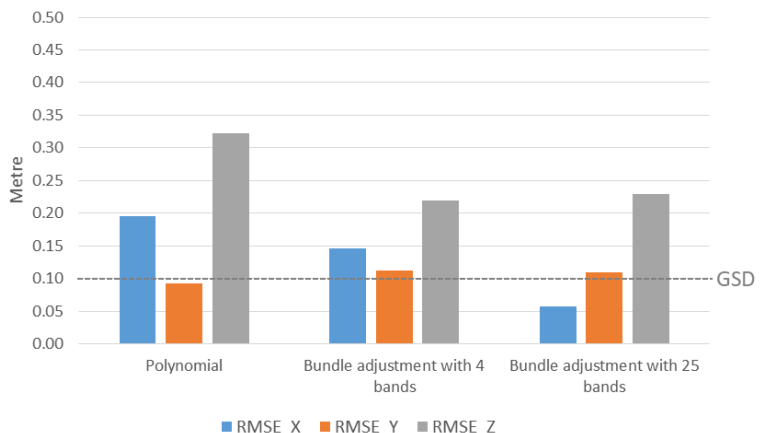

Figure 5. RMSE at checkpoints considering image orientation with Rikola navigation data.

Figure 6 presents the RMSE resulting in the checkpoints when the initial camera positions were provided by the NavSpark GPS receiver. The bundle adjustment with 25 bands resulted in discrepancies of approximately $10 \mathrm{~cm}$ in XY (1 GSD) and $13.2 \mathrm{~cm}$ in $\mathrm{Z}$ (<1.5 GSD). When only four bands were considered, the discrepancies were slightly larger than 1 GSD, approximately $11 \mathrm{~cm}$ in XY and 2 GSD in Z (X GSD). With the polynomial technique, results closer to the 25-band bundle adjustment trial were obtained, since the RMSEs were less than $10 \mathrm{~cm}$ in $\mathrm{XY}(<1 \mathrm{GSD})$ and $18.9 \mathrm{~cm}$ in $\mathrm{Z}(<2 \mathrm{GSD})$.

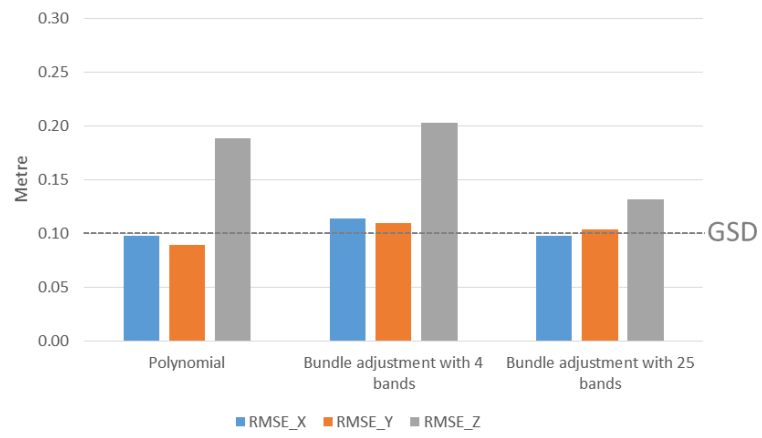

Figure 6. RMSE at checkpoints considering image orientation with initial camera positions provided by the NavSpark GPS receiver.

The graph in Figure 7 shows the mean of the differences between the initial camera positions provided by the NavSpark GPS and the positions XYZ resulting from the polynomial and conventional bundle adjustment techniques. The polynomial technique indicated an average difference of approximately 14 $\mathrm{cm}$ in $\mathrm{X}, 21 \mathrm{~cm}$ in $\mathrm{Y}$ and $26 \mathrm{~cm}$ in Z, which were smaller than those differences obtained by the two bundle adjustments trials, except in $\mathrm{Z}$ coordinate using the bundle adjustment with 25 bands. When using all bands, a larger number of rays provides a better estimation of elevations (Z). This effect can also be observed by comparing the two bundle adjustment trials (with 4 and 25 bands): using 4 bands resulted in estimated values with larger errors. With respect to the polynomial technique, the estimated $\mathrm{Z}$ values achieved intermediate differences, when comparing to the two bundle adjustments. This happened because the polynomial technique imposed a mathematical model of trajectory to which the observations must fit.

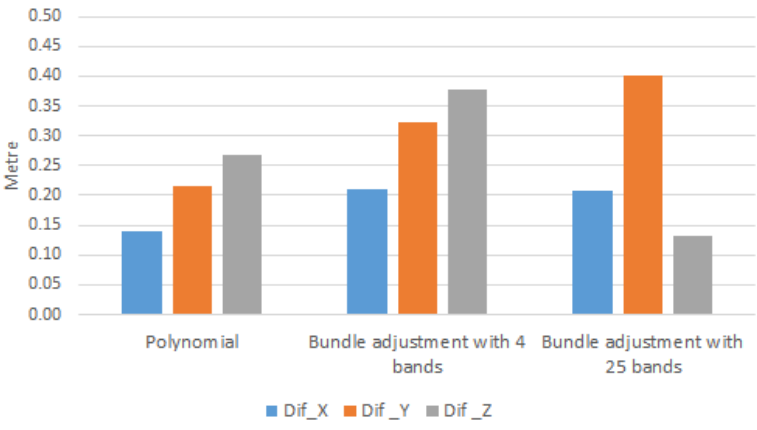

Figure 7. Mean of differences calculated between the NavSpark trajectory and the XYZ positions estimated by the image orientation techniques.

Figures $8(\mathrm{a}, \mathrm{b})$ exemplify the behaviour of 25 bands positons within a hypercube. The band displacement is graphically presented using positions determined by three techniques: NavSpark GPS, bundle adjustment with 25 bands and polynomial technique. As it can be seen, the camera positions acquired by the NavSpark GPS follow an approximately linear trajectory, which is preserved when the EOPs are estimated by the polynomial technique, that is, the observations are adjusted to the trajectory model to estimate the polynomial parameters. Although the bundle adjustment yields accurate results, the trajectory not recovered because the errors are distributed by least squares adjustment without the geometric constraint.
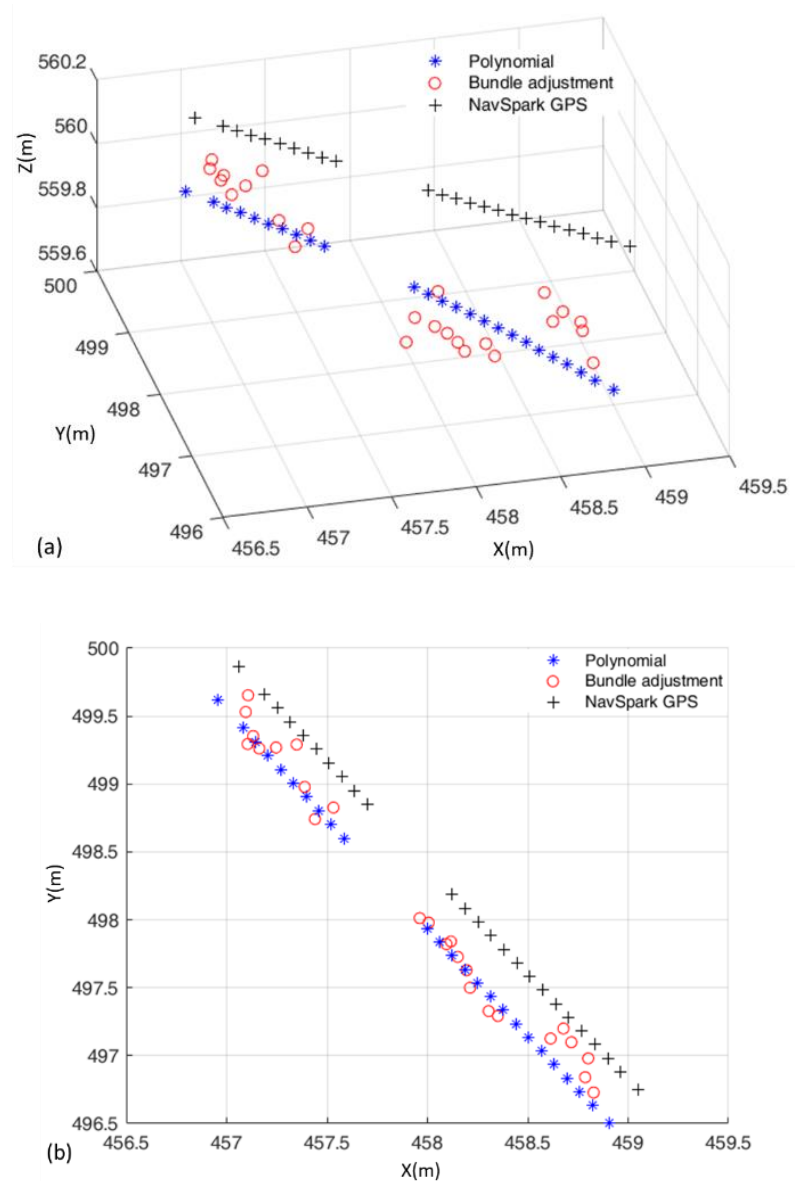

Figure 8. Bands trajectories in a hypercube resulting from three techniques: (a) isometric view and (b) 2D top view. 
Comparing bundle adjustment and polynomial technique, the results of the adjustment in $\mathrm{Z}$ indicated a random behavior in the sequence of bands, as shown in Figure 8(a). On the other hand, in Figure 8(b), the band displacement presented similarity in relation to the planimetry. As for the NavSpark GPS positions, the two orientation techniques converged on the same side of displacement, both in planimetry and in altimetry. It is important to note that such displacements may have been influenced by the IOPs, which depend on a precise modeling of the air gap variations in the interferometer. However, the discrepancies assessed at the checkpoints indicated that the results achieved an accuracy of 1 GSD in XY and less than 2 GSD in Z, demonstrating the potential of the polynomial technique.

\section{CONCLUSIONS}

This study presented experiments on the EOP estimation using polynomial models applied to a hyperspectral frame camera with time-sequential acquisition. The main objective was to evaluate the performance of the polynomial approach when estimating EOPs of UAV images. The experiments considered 34 hypercubes in two flight strips acquired with a navigation GPS integrated into the camera and precision single frequency NavSpark GPS receiver. The polynomial technique was applied to four sample bands to estimate parameters of polynomial functions and subsequently to determine the EOPs of the other bands within each hypercube, completing 25 bands.

Although the polynomial technique has been able to estimate the EOPs of hypercubes using positions from navigation data, as expected, the results were better with camera positions provided by the NavSpark GPS, due to better data accuracy. When comparing with the conventional bundle adjustment including all bands, the polynomial technique obtained similar results in planimetry, which were approximately 1 GSD. In altimetry, although the bundle adjustment has produced a smaller discrepancy, the polynomial technique also resulted in a discrepancy less than 2 GSDs. The differences in $\mathrm{Z}$ between the two techniques were $1 / 2$ GSD. Thus, such results demonstrated that the approach with polynomials can also produce accurate results, which yields benefits because it requires few bands for the image orientation procedure. Additionally, less control point measurement and tie point editing are required.

However, further studies are still need to improve the determination of boresight angles and the estimation of IOPs. The effects of the interferometer on spectral band acquisition still need to be further investigated to enable a more refined modelling, which can increase the accuracy level of the polynomial technique.

\section{ACKNOWLEDGEMENTS}

The authors would like to thank the National Research Council (CNPq) [grants 307554/2014-7, 404379/2016-8, 150306/2018-0] and the São Paulo Research Foundation (FAPESP) [grants 2013/50426-4, 2014/05533-7] for financial support.

\section{REFERENCES}

Aasen, H., Burkart, A., Bolten, A., Bareth, G., 2015. Generating 3D hyperspectral information with lightweight UAV snapshot cameras for vegetation monitoring: From camera calibration to quality assurance. ISPRS Journal of Photogrammetry and $\begin{aligned} & \text { Remote } \\ & \text { Sensing }\end{aligned}$ 108,
doi.org/10.1016/j.isprsjprs.2015.08.002

Berveglieri, A., Tommaselli, A.M.G., Honkavaara, E., 2017. Estimating exterior orientation parameters of hyperspectral bands based on polynomial models. ISPRS - International Archives of the Photogrammetry, Remote Sensing and Spatial Information Sciences XLII-3/W3, 19-25. doi.org/10.5194/isprsarchives-XLII-3-W3-19-2017.

Fryer, J.G., Brown, D.C., 1986. Lens Distortion for Close-Range Photogrammetry. Photogrammetric Engineering and Remote Sensing 52, 51-58.

Garrido-Jurado, S., Muñoz-Salinas, R., Madrid-Cuevas, F.J., Marín-Jiménez, M.J., 2014. Automatic generation and detection of highly reliable fiducial markers under occlusion. Pattern Recognition 47, 2280-2292. doi.org/10.1016/j.patcog.2014.01.005

Honkavaara, E., Rosnell, T., Oliveira, R., Tommaselli, A., 2017. Band registration of tuneable frame format hyperspectral UAV imagers in complex scenes. ISPRS Journal of Photogrammetry and Remote Sensing 134, 96-109. doi.org/10.1016/j.isprsjprs.2017.10.014.

Kenefick, J.F., Gyer, M.S., Harp, B.F., 1972. Analytical selfcalibration. Photogrammetric Engineering 38, 1117-1126.

Marcato Junior, J., Tommaselli, A.M.G., 2013. Exterior orientation of CBERS-2B imagery using multi-feature control and orbital data. ISPRS Journal of Photogrammetry and Remote Sensing 79, 219-225. doi.org/10.1016/j.isprsjprs.2013.02.018.

Nevalainen, O., Honkavaara, E., Tuominen, S., Viljanen, N., Hakala, T., Yu, X., Hyyppä, J., Saari, H., Pölönen, I., Imai, N.N., Tommaselli, A.M.G., 2017. Individual Tree Detection and Classification with UAV-Based Photogrammetric Point Clouds and Hyperspectral Imaging. Remote Sensing 9. doi.org/10.3390/rs9030185.

Oliveira, R.A., Tommaselli, A.M.G., Honkavaara, E., 2019. Generating a hyperspectral digital surface model using a hyperspectral 2D frame camera. ISPRS Journal of Photogrammetry and Remote Sensing 147, 345-360. https://doi.org/10.1016/j.isprsjprs.2018.11.025.

Oliveira, R.A., Tommaselli, A.M.G., Honkavaara, E., 2016. Geometric calibration of a hyperspectral frame camera. $\begin{array}{llll}\text { Photogrammetric } \quad \text { Record 325-347. } & \text { 31, }\end{array}$ doi.org/10.1111/phor.12153.

Saari, H., Pellikka, I., Pesonen, L., Tuominen, S., Heikkilä, J., Holmlund, C., Mäkynen, J., Ojala, K., Antila, T., 2011. Unmanned aerial vehicle (UAV) operated spectral camera system for forest and agriculture applications, in: Proceedings of SPIE.

Senop Ltd., 2017. . Hyperspectral camera. URL http://senop.fi/en/optronics-hyperspectral (accessed 2.12.18).

Tommaselli, A.M.G., Galo, M., Moraes, M.V.A., Marcato Junior, J., Caldeira, C.R.T., Lopes, R.F., 2013. Generating virtual images from oblique frames. Remote Sensing 5, 1875-1893. 